Известия НАН Армении, Физика, т.57, №1, с.141-148 (2022)

УДК 602

DOI:10.54503/0002-3035-2022-57.1-141

\title{
ДИНАМИКА ЗАРЯЖЕННЫХ СЛОЖНЫХ МОЛЕКУЛ В ВОДНОМ РАСТВОРЕ
}

\author{
Ф.В. ГАСПАРЯН ${ }^{1 *}$, Л.Ф. ГАСПАРЯН ${ }^{1,2}$, В.В. СИМОНЯН ${ }^{2}$ \\ ${ }^{1}$ Ереванский государственный университет, Ереван, Армения \\ ${ }^{2}$ DNA-HIVE LLC, Rockville, MD, USA \\ *e-mail: fgaspar@ysu.am
}

(Поступила в редакцию 12 октября 2021 г.)

\begin{abstract}
Представлены результаты теоретического моделирования динамики заряженной большой молекулы в водном растворе под влиянием силы тяжести, направляющего электрического поля и выталкивающей силы. Расчеты проведены на примере ДНК молекулы. Показано, что электрическим полем можно плавно регулировать скорость движения молекулы так, чтобы можно было провести точное/медленнее секвенирования методом туннельного тока. Показано также, что скорость движения молекулы можно уменьшить до нескольких десятичных см/с, что равносильно секвенированию $10^{5}-10^{6}$ нуклеотидов в секунду.
\end{abstract}

\section{1. Введение}

С момента открытия спиральной структуры дезоксинуклеиновой кислоты (ДНК) [1] исследования ДНК-сенсоров резко расширились. Процесс секвенирования ДНК - это точное определение количества и распределения нуклеотидов (А-аденин, Т-тимин, С-цитозин и G-гуанин) в молекулах ДНК. Одна из задач секвенирования - создание надежных, стабильных и воспроизводимых наноразмерных устройств. Для высокопроизводительной идентификации последовательностей одномолекулярных нуклеиновых кислот были предложены несколько наноразмерных электронных методов.

Под действием электрического поля заряженные нуклеотиды могут захватываться и проходить через канал или пору в процессе, называемом «транслокацией». За последние десятилетия поры в масштабе одного нанометра продемонстрировали отличную способность обнаруживать, идентифицировать и характеризовать различные аналиты и биомолекулы, такие как бактерии [2], белки [3-7], антитела [8,9], нуклеиновая кислота [10,11], ДНК [12,13], РНК $[14,15]$.

Известны следующие методы электрического обнаружения секвенирования ДНК на основе нанопор:

- метод основанный на блокаде ионного тока $[12,16,17]$;

- метод основанный на туннельном токе [18-22]. 
Одним из потенциальных методов будущего является секвенирование ДНК туннельными токами [23]. В методике секвенирования ДНК туннельным током, она проходит через пору или канал. При прохождении каждой базы ДНК протекают достаточно разные туннельные токи, а последовательность базовых сигналов выводиться из различий в туннельных токах. При этом методе секвенирования может происходить на порядки быстрее, чем при методе блокады ионного тока $[23,24]$, а туннельный ток на несколько порядков выше, чем ионный.

В последние годы были достигнуты большие успехи, и были предложены многие конструкции для новых подходов к биомолекулярному зондированию с использованием наноэлектроники, включая появление туннельных переходов в качестве сенсорной платформы. Наноразмерные электроды привлекли большое внимание из-за их потенциального применения в прогрессивной миниатюризации электроники и в качестве наноразмерного инструмента для анализа свойств в молекулярном масштабе. Электроды с зазором можно просто определить как пару электродов с зазором, который может измеряться в нанометрах. Транспортная динамика заряженных молекул в наноразмерных порах, расположенных в биожидкостях, является результатом множества факторов, включая гидродинамические и электростатические взаимодействия, а также диффузию.

Было предложено секвенировать отдельные молекулы ДНК путем измерения физических свойств оснований при их прохождении через нанопоры [25]. Расчеты показывают, что электронное туннелирование может идентифицировать основания в оцДНК без ферментативного процессинга [25-27], и было экспериментально показано, что туннелирование может распознавать отдельные нуклеотиды $[28,29]$ и короткие олигомеры ДНК [30]. Графеновые нанощели и нанопоры также обладают потенциалом для электрического секвенирования ДНК [31]. Метод механически управляемых обрывных переходов (MCBJ, Mechanically Controllable Break Junction) успешно используется для исследования транслокационного поведения молекул ДНК [19,26,28].

Проведенные нами недавно расчеты показали ээффективность метода туннельного тока через наноразмерный щель в процессе регулирования скорости движения, значит и скорости секвенирования ДНК молекулы в водном растворе [32].

В настоящей работе предложен альтернативный подход теоретического моделирования скорости движения ДНК молекулы в водном растворе.

\section{2. Динамика движения молекулы}

Схематическая картина ячейки для исследования динамики движения заряженной молекулы в водном растворе представлена на рис.1. Считается, что молекула заряжена отрицательно, типично для молекулы ДНК. Секвенирование молекулы производится методом туннельного тока. С этой целью в измерительной ячейке «смонтированы» изолированные перпендикулярно движению ДНК 


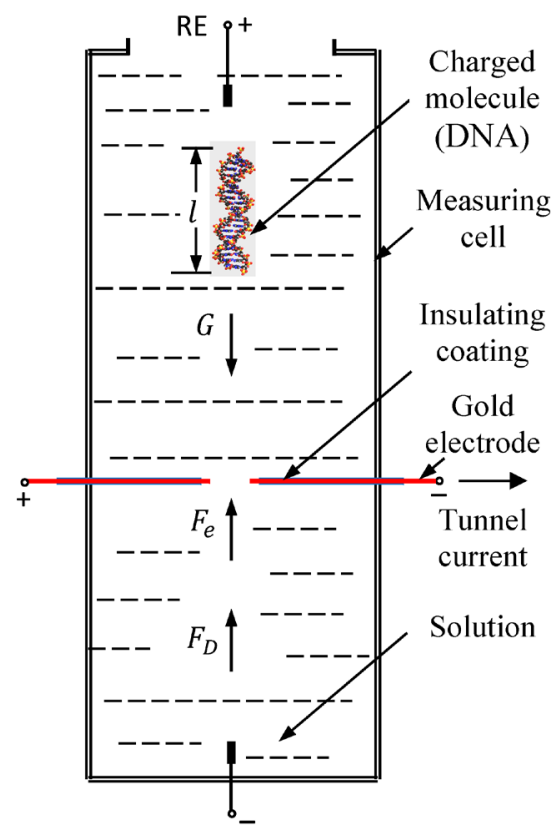

Рис.1. Схематическая картина измерительной ячейки для исследования динамики движения заряженной молекулы в водном растворе под действием сил тяжести, сопротивления и электрического поля.

золотые электроды с наноразмерным зазором (например, методом МСВJ).

Для идентификации неизвестной молекулы будем использовать метод основанная на измерении туннельного тока через молекулу [18-21].

Движения молекулы в водном растворе определяется силой тяжести $\mathbf{G}$ и силой сопротивления (отталкивания) $\mathbf{F}_{D}$. Для регулирования скорости вертикального движения заряженной молекулы $v$ используем также дополнительную силу электрического поля $\mathbf{F}_{E l}$, создаваемой потенциалом приложенным к электроду сравнения RE. Тогда результирующая сила $\mathbf{F}_{\text {Tot }}$, действующая на молекулу массой $m$ будет ровна:

$$
\mathbf{F}_{T o t}=\mathbf{G}+\mathbf{F}_{E l}+\mathbf{F}_{D} .
$$

Под этой силой молекула будет двигаться со скоростью v:

$$
\mathbf{G}+\mathbf{F}_{E l}+\mathbf{F}_{D}=m \frac{\partial \mathbf{v}}{\partial t}, \quad m g \pm e E-\frac{1}{2} C_{D} \rho v^{2} A=m \frac{\partial v}{\partial t} .
$$

Здесь $g$ ускорение свободного падения молекулы в водной среде, которое принимается равной $9.8 \mathrm{~m} / \mathrm{s}^{2}, \rho$ — плотность водного раствора $\rho=3.446 \delta \times 10^{-3}+$ $1.0048 \mathrm{~g} / \mathrm{ml}=10^{3} \mathrm{~kg} / \mathrm{m}^{3}$ [33], где $\delta$ процентный состав. Плотность водного раствора изменяется очень мало, например при $0<\delta \leq 20,1<\rho \leq 1.1 \mathrm{~g} / \mathrm{ml}, C_{D}$ коэффициент сопротивления - безразмерное число, определяемое сечением молекулы (при полусферической поверхности $C_{D} \approx 0.42$ [34]), $A$ - площадь поперечного сечения молекулы

$$
A=\frac{\pi d^{2}}{4} \approx \frac{3.14 \times 2.2^{2}}{4} \mathrm{~nm}^{2}=3.8 \times 10^{-18} \mathrm{~m}^{2},
$$


$d \approx 2.2 \mathrm{~nm}$ - диаметер молекулы ДНК [35].

Масса молекулы $m$ монжо представить как сумма масс отдельных блоков нуклеотидов аденина, тимина, цитозина и гуанина $m_{n}$ :

$$
m=m_{n} N_{n} .
$$

Здесь $N_{n}$ - число групп нукеизидов в молекуле ДНА, $m_{n}=8 \times 10^{-25}$ кг [32]. Принимая $N_{n}=10^{6}$, получим $m=8 \times 10^{-19} \mathrm{~kg}$.

Введем следующие обозначения:

$$
B_{1} \equiv \frac{1}{2 m} C_{D} \rho A, \quad B_{2} \equiv g \pm \frac{e E}{m} .
$$

Тогда из (2) имеем

$$
\frac{\partial v}{\partial t}=B_{2}-B_{1} v^{2}, \frac{d v}{B_{2}-B_{1} v^{2}}=d t
$$

Интегрируя полуденное уравнение от $v_{0}$ до $v$, получим

$$
\int_{v_{0}}^{v} \frac{d v}{B_{2}-B_{1} v^{2}}=\int_{t_{0}}^{t} d t
$$

Решение ур. (5) имеет вид:

$$
\frac{1}{\sqrt{B_{1} B_{2}}} \arctan \sqrt{\frac{B_{1}}{B_{2}}}\left(v-v_{0}\right)=t-t_{0} .
$$

Здесь $v_{0}$ начальная скорость молекулы в момент движения $t_{0}, v$-конечная скорость в момент времени $t$.

Разложим в ряд функцию $\arctan x$

$$
\arctan x=x-\frac{x^{3}}{3}+\frac{x^{5}}{5}
$$

при $x^{2}<1$ или

$$
\frac{B_{1}}{B_{2}}\left(v-v_{0}\right)^{2}<1 .
$$

Ясно, что для точной идентификации исследуемой молекулы (в случае ДНК молекулы - для точного секвенирования) необходимо увеличить время «удержания» молекул между латеральными электродами, чтобы «успеть» измерить слабый туннельный ток (рис.1). Для этого необходимо уменьшить скорость вертикального движения молекулы и перед электрической силой в (2) использовать знак «-». Тогда условие (8) будет иметь вид:

$$
\frac{C_{D} \rho A}{m g-e E}<1
$$

Сохраняя первые два члена ряда в (7), для скорости молекулы получим кубическое уравнение:

$$
\frac{1}{\sqrt{B_{1} B_{2}}}\left\{\sqrt{\frac{B_{1}}{B_{2}}}\left(v-v_{0}\right)-\frac{1}{3}\left[\sqrt{\frac{B_{1}}{B_{2}}}\left(v-v_{0}\right)\right]^{3}+\ldots\right\}=t-t_{0} .
$$

Обозначая

$$
\left(v-v_{0}\right) \equiv y, \quad p \equiv-\frac{3 B_{2}}{B_{1}}, q \equiv \frac{3 B_{2}^{2}\left(t-t_{0}\right)}{B_{1}},
$$

получим кубическое уравнение канонической формы

$$
y^{3}+p y+q=0 \text {. }
$$


По формуле Кардано корни кубического уравнения канонической формы (11) равны:

$$
\begin{gathered}
y_{1}=\alpha+\beta \\
y_{2,3}=-\frac{\alpha+\beta}{2} \pm i \frac{\alpha-\beta}{2} \sqrt{3} \\
\alpha=\sqrt[3]{-\frac{q}{2}+\sqrt{Q}}, \quad \beta=\sqrt[3]{-\frac{q}{2}-\sqrt{Q}}, \quad Q=\left(\frac{p}{3}\right)^{3}+\left(\frac{q}{2}\right)^{2} .
\end{gathered}
$$

Так как скорость движения ДНК молекулы не может быть мнимой или отрицательной величиной, то в дальнейшем будем использовать решение (12a):

$$
y=y_{1} \equiv v-v_{0}=\sqrt[3]{-\frac{q}{2}+\sqrt{\left(\frac{p}{3}\right)^{3}+\left(\frac{q}{2}\right)^{2}}}+\sqrt[3]{-\frac{q}{2}-\sqrt{\left(\frac{p}{3}\right)^{3}+\left(\frac{q}{2}\right)^{2}}} .
$$

Так как $v$ реальная величина, и учитывая, что $p<0$, в правой части (14) оставим только второй корень и разложим в нем квадратный корень. Получим

$$
v=v_{0}+\frac{B_{2}}{B_{1}}\left[1-\frac{1}{3 B_{1} B_{2}\left(t-t_{0}\right)^{2}}\right] \sqrt[3]{\frac{B_{1}^{2}\left(t-t_{0}\right)}{9 B_{2}}} .
$$

При разложении корня должно выполнятся условие

$$
1>\frac{1}{2}\left(\frac{2}{q}\right)^{2}\left(\frac{p}{3}\right)^{3}
$$

что равносильно условию

$$
E<E_{c r}=\frac{m g}{e}-\frac{4 m^{2}}{9 C_{D} \rho A t^{2}} .
$$

Критическое значение поля $E_{c r}$ определяется условием $v=0$. Принимая, что в начальный момент движения $\left(t_{0}=0\right)$ начальная скорость молекулы ровна нулью $\left(v_{0}=0\right)$, окончательно получим:

$$
v=\frac{2(m g-e E)}{C_{D} \rho A}\left[1-\frac{2 m^{2}}{3 C_{D} \rho A(m g-e E) t^{2}}\right] \sqrt[3]{\frac{\left(C_{D} \rho A\right)^{2} t}{36 m(m g-e E)}} .
$$

Скорость движения молекулы зависит как от параметров самой молекулы $\left(m, A, C_{D}\right)$, так и от внешних параметров $(E, \rho)$.

\section{3. Обсуждение результатов}

Напомним, что $t$ в формулах (16) и (17) представляет собой время действия внешнего направляющего электрического поля $E$, то есть время, прошедшее после смешивания ДНК молекулов (или капли крови) пациента с водным раствором. Ускоряясь за время $t$ под действием результирующей силы, молекула в водном растворе достигает скорости $v$ и с такой скоростью входит в межэлектродную щель. При подачи импульсов на латеральные электроды создается «кратковременный» молекулярный переход. Под приложенным к поперечному золотому электроду потенциала через этот переход протекает туннельный ток, величина которой характеризует данная база молекулы. Начинается секвенирование ДНК молекулы. 


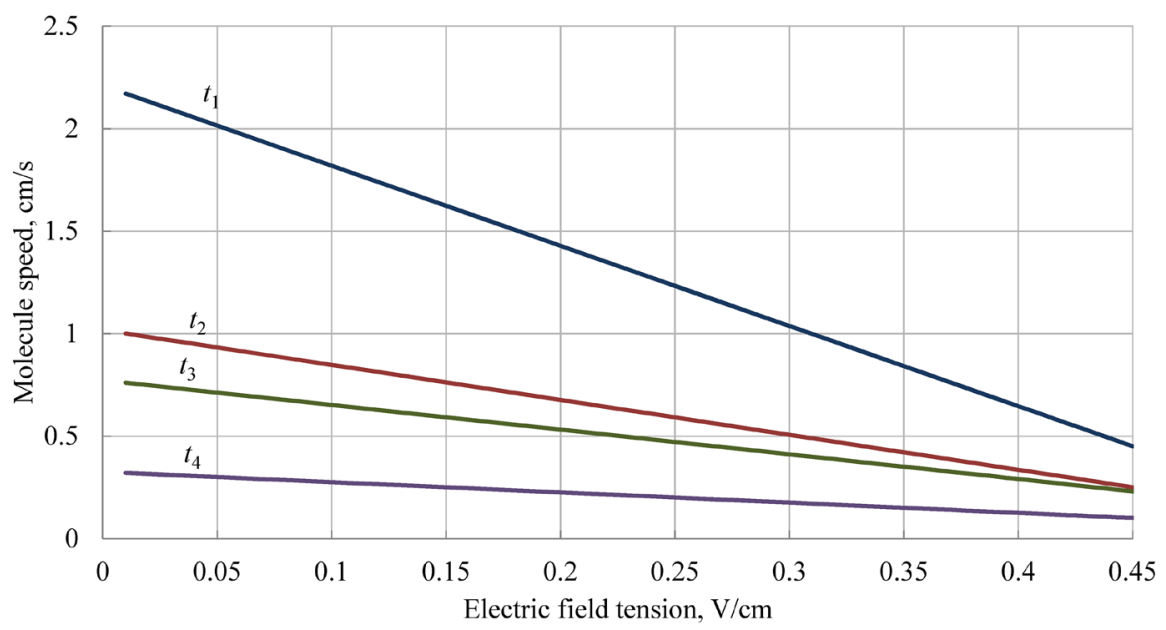

Рис.2. Зависимость скорости молекулы ДНК от напряженности регулирующего внешнего поля: Кривые соответствуют случаям: $t_{1}=1 \mathrm{~s}, t_{2}=$ $0.1 \mathrm{~s}, t_{3}=0.05 \mathrm{~s}, t_{4}=0.01 \mathrm{~s}$.

На рис. 2 представлена зависимость скорости молекулы от величины регулирующего электрического поля $E$ используя значения постоянных и принимая, что количество блоков из четырех баз ДНА молекулы $N_{n}=10^{6}$, масса одного блока $m_{n}=8 \times 10^{-25} \mathrm{~kg}$, диаметр и площадь поперечного сечения ДНА молекулы соответственно равны $d \approx 2.2 \mathrm{~nm}$ и $A=3.8 \times 10^{-18} \mathrm{~m}^{2}$.

Для сравнения полученных данных, в табл. 1 приведены известные из литературы данные о скорости чтения/движения ДНА молекулы. Считая, что длина базы порядка $1 \mathrm{~nm}$ [32], скорость чтения в единицах base/s лучше поменять на $\mathrm{nm} / \mathrm{s}$.

Табл.1.

\begin{tabular}{|c|c|c|c|c|}
\hline $\begin{array}{c}\text { Скорость чтения ДНК } \\
\text { молекулы, base/s, nm/s }\end{array}$ & 70000000 & 55000 & 9600 & $10-1000$ \\
\hline Литература & {$[36]$} & {$[37]$} & {$[38]$} & {$[39]$} \\
\hline
\end{tabular}

С ростом вертикального электрического поля скорость движения молекулы замедляется. Критическое значение этого поля $E_{c r}$ ограничена останавлением движенея молекулы и определяется условием (17). При использованных нами выше параметрах $E_{c r}=0.5 \mathrm{~V} / \mathrm{cm}$.

С уменьшением времени воздействия электрического поля $t$ скорость движения молекулы замедляется.

\section{4. Заключение}

С учетом сил тяжести, отталкивания и силы внешнего электрического поля моделирован динамика заряженной сложной молекулы в водном растворе. Установлено, что можно эффективно регулировать скоростью движения молекулы в 
водной среде манипулируя внешним электрическим полем и временем его воздействия. Численные оценки, проведенные для случая ДНК молекулы, показывают, что полученные значения скорости сильно не отличаются от известных литературных данных. Можно плавно регулировать скорость чтения ДНК молекулы и провести точное секвенирование. Показано также, что скорость движения молекулы можно уменьшить до нескольких десятичных $\mathrm{cm} / \mathrm{s}$, что равносильно секвенированию $10^{5} \div 10^{6}$ нуклеидов в секунду.

\section{ЛИТЕРАТУРА}

1. J.D. Watson, F.H.C. Crick. Nature, 171, 737 (1953).

2. N.J. Loman, J. Quick, J.T. Simpson. Nat. Methods, 12, 733 (2015).

3. J. Clarke, H.-C. Wu, L. Jayasinghe, et al. Nat. Nanotechnol., 4(4), 265 (2009).

4. C. Plesa, J.W. Ruitenberg, M.J. Witteveen, C. Dekker. Nano Lett., 15, 3153 (2015).

5. E. Kennedy, Z. Dong, C. Tennant, G. Timp. Nat. Nanotechnol., 11, 968 (2016).

6. X. Lin, A.P. Ivanov, J.B. Edel. Chem. Sci., 8, 3905 (2017).

7. C. Wloka, V. Van Meervelt, D. van Gelder, et al. ACS Nano, 11, 4387 (2017).

8. S. Wang, F. Haque, P.G. Rychahou, B.M. Evers, P. Guo. ACS Nano., 7, 9814 (2013).

9. A.B. Farimani, M. Heiranian, K. Min, N.R. Aluru. J. Phys. Chem. Lett., 8, 1670 (2017).

10. Y.-L. Ying, J. Zhang, R. Gao, Y.-T. Long. Angew. Chem., Int. Ed., 52, 13154 (2013).

11. F. Sanger, S. Nicklen, A.R. Coulson. Proc. Natl. Acad. Sci. U.S.A., 74(12), 5463 (1977).

12. S. Howorka, S. Cheley, H. Bayley. Nat. Biotechnol., 19, 636 (2001).

13. M. Rincon-Restrepo, E. Mikhailova, H. Bayley, G. Maglia. Nano Lett., 11, 746 (2011).

14. M. Clamer, L. Höfler, E. Mikhailova, G. Viero, H. Bayley. ACS Nano., 8, 1364 (2014).

15. O.K. Zahid, F. Wang, J.A. Ruzicka, E.W. Taylor, A.R. Hall. Nano Lett., 16, 2033 (2016).

16. T.Z. Butler, J.H. Gundlach, M. Troll. Biophys. J., 93(9), 3229 (2007).

17. K. Chen, M. Juhasz, F. Gularek, E. Weinhold, et al. Nano Lett., 17(9), 5199 (2017).

18. J. He, L. Lin, P. Zhang, S. Lindsay. Nano Lett., 7(12), 3854 (2007).

19. M. Taniguchi. In: 18th Int. Conf. on Miniaturized Systems for Chemistry and Life Sciences, October 26-30, 2014, San Antonio, Texas, USA, pp.199-204, 2014.

20. M. Xu, R.G. Endres, Y. Arakawa. Small, 3(9), 1539 (2007).

21. M. Zwolak, M.Di. Ventra. Nano Letters, 5(3), 421 (2005).

22. US FDA, United States Food and Drug Administration. Nucleic Acid Based Tests (2016).

23. W.J. Ansorge. Next Generat .Sequenc. \& Applic. S1:005, 1 (2016).

24. L. Gasparyan, I. Mazo, V. Simonyan, F. Gasparyan. J. Contemp. Phys., 55, 77 (2020).

25. D. Branton, D.W. Deamer, A. Marziali, et al. Nature Biotechnol., 26, 1146 (2008).

26. P.S. Krstic, J.C. Wells, M. Fuentes-Cabrera, D. Xu, J.W. Lee. Solid State Phenom., 121, 1387 (2007).

27. J. Lagerqvist, M. Zwolak, M. Di Ventra. Biophys. J., 93, 2384 (2007).

28. M. Tsutsui, M. Taniguchi, K. Yokota, T. Kawai. Nature Nanotech., 5, 286 (2010).

29. S. Chang, S. Huang, J. He, F. Liang, et al. Nano Lett., 10, 1070 (2010).

30. S. Huang, J. He, S. Chang, P. Zhang, et al. Nature Nanotechnology, 5, 868 (2010).

31. J. Prasongkit, A. Grigoriev, B. Pathak, et al. J. Phys. Chem. C, 117(29), 15421 (2013). 
32. L. Gasparyan, I. Mazo, V. Simonyan, F. Gasparyan. Open Journal of Biophysics, 10, 96 (2020).

33. P. Chassaing, R.A. Antonia, F. Anselmet, L. Joly, S. Sarkar. Fluid Mechanics and its Applications. vol. 69. Variable Density Fluid Turbulence. Springer Netherlands, 2002.

34. B.W. McCormick. Aerodynamics, Aeronautics, and Flight Mechanics. p. 24, John Wiley \& Sons, Inc., New York, 1979.

35. M. Mandelkern, J.G. Elias, D. Eden, D.M. Crothers. Journal of Molecular Biology, 152(1), 153 (1981).

36. A. Smolyanitsky, B.I. Yakobson, et al. ACS Nano, 10(9), 9009 (2016).

37. Y. He, M. Tsutsui, C. Fan, M. Taniguchi, et al. ACS Nano, 5(7), 5509 (2011).

38. H.B. Peng, X.S.S. Ling. Nanotechnology, 20(18), 185101 (2009).

39. D. Fologea, J. Uplinger, B. Thomas, et al. Nano Letters, 5(9), 1734 (2005).

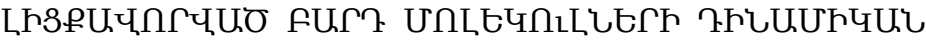

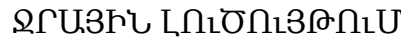

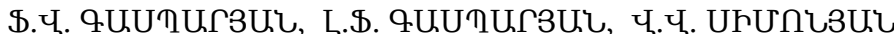

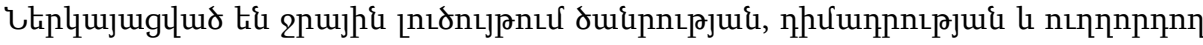

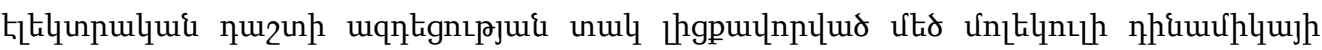

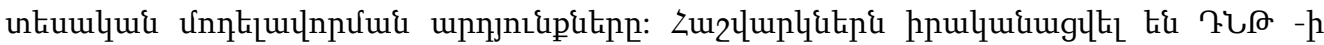

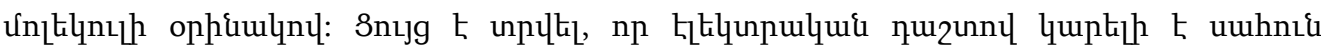

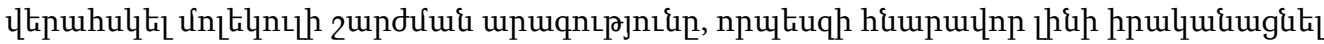

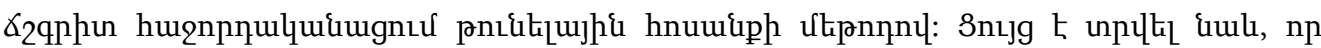

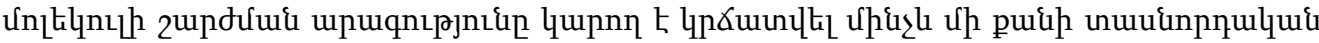

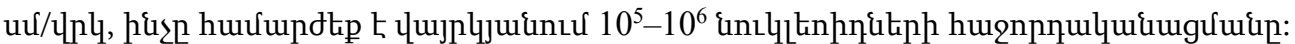

\section{DYNAMICS OF CHARGED COMPLEX MOLECULES IN AQUEOUS SOLUTION}

\section{F.V. GASPARYAN, L.F. GASPARYAN, V.V. SIMONYAN}

The results of theoretical modeling of the dynamics of a charged large molecule in an aqueous solution under the influence of gravity, vertical electric field and drag force are presented. Calculations were carried out for the DNA molecule. It has been shown that an electric field can smoothly control the speed of movement of a molecule so that it is possible to carry out accurate sequencing by the tunneling current method. It was also shown that the speed of movement of a molecule can be reduced to several decimal $\mathrm{cm} / \mathrm{s}$, which is equivalent to sequencing $10^{5}-10^{6}$ nucleoids per second. 